\title{
Manejo Conservador del Trauma Penetrante de Tórax, Casuística de 2 Años en Hospital Universitario del Valle en Cali, Colombia, un Importante Centro de Trauma
}

\author{
${ }^{1}$ Raúl Barros Gruezo, ${ }^{2}$ Jair Díaz Martínez
}

\section{RESUMEN}

El traumatismo torácico representa uno de los mayores desafíos en el manejo integral del paciente politraumatizado debido a la alta morbilidad y mortalidad generada por sus complicaciones y los altos costos de la atención de salud. La mayoría de las lesiones torácicas son manejadas con maniobras simples como el tubo de toracostomía, apenas el 10 al $15 \%$ de los pacientes necesitan tratamiento quirúrgico. El hemo-neumotórax es la lesión más comúnmente encontrada en el trauma de tórax con una prevalencia del $52.3 \%$, seguido por el Hemotórax $23.4 \%$ y el neumotórax del $20 \%$ de los casos. Hasta el $30-80 \%$ de los pacientes con lesiones traqueobronquiales fallecen en el lugar del accidente. Este estudio de colección retrospectiva de datos incluyo a los pacientes del registro de trauma, que sufrieron trauma penetrante de tórax en un periodo de 2 años y que fueron atendidos en el hospital universitario del valle, encontrándose 587 pacientes con este tipo de trauma, con un total de pacientes operados 257 de los cuales 224 fueron dados de alta y 33 fallecieron; de los 330 pacientes que no fueron operados 262 egresaron y 62 murieron. La mayoría eran hombres 557 y mujeres 30; con 2 tipos de heridas 247 punzocortantes y 340 heridas por proyectil de arma de fuego. Esta importante casuística de las pocas en el mundo, nos permite analizar que el trauma penetrante de tórax es mortal dependiendo de la estructura comprometida y que sin embargo es factible realizar manejo conservador en pacientes seleccionados; no guarda relación a las estadísticas mundiales pues los pacientes operados rebasan el $15 \%$, la importancia radica en que el volumen de pacientes muy graves con necesidad quirúrgica es muy grande por lo que es prioritario identificar quienes se beneficiarían de manejo conservador.

Palabras claves: Casuística importante de trauma de tórax, Manejo conservador del trauma de tórax, Trauma penetrante de tórax.

\section{${ }^{1,2}$ Residente De Cirurgia-Laparoscopia \\ ${ }^{1}$ Universidad Internacional del Ecuador, Hospital Metropolitano Quito, Ecuador \\ ${ }^{2}$ Universidad Nacional Autónoma De México, Hospital General Tacuba, México DF, México}

Corresponding Author: Raúl Barros Gruezo, Residente De Cirurgía-Laparoscopia, Universidad Internacional Del Ecuador, Hospital Metropolitano Quito, Ecuador, e-mail: raulbarros2006@yahoo.com
How to cite this article: Gruezo RB, Martínez JD. Manejo Conservador del Trauma Penetrante de Tórax, Casuística de 2 Años en Hospital Universitario del Valle en Cali, Colombia, un Importante Centro de Trauma. Panam J Trauma Crit Care Emerg Surg 2015;4(3):188-193.

Source of support: Nil

Conflict of interest: None

\section{ABSTRACT}

Chest injury represents one of the greatest challenges in the comprehensive management of trauma patients because of the high morbidity and mortality caused by complications and high costs of healthcare. Most chest injuries are managed by simple maneuvers such as tube thoracostomy, only 10 to $15 \%$ of patients need surgical treatment. Hemopneumothorax is most commonly found in chest trauma with a prevalence of $52.3 \%$, followed by $23.4 \%$ hemothorax and pneumothorax in $20 \%$. Up to 30 to $80 \%$ of patients with tracheobronchial injury die at the scene. This study of a retrospective collection of data included patients from trauma registry, who suffered penetrating chest trauma over a period of 2 years and were treated at the university hospital. A total of 587 patients had this type of trauma, a total of 257 were operated, of which 224 were discharged and 33 died; of the 330 patients who were not operated 262 were discharged and 62 died. Most were men; 247 were injured by gun fire. Penetrating chest trauma is fatal depending on the compromised structure and yet conservative management is feasible in selected patients. As against global statistics, operated cases exceed $15 \%$. The importance is that the volume of critically ill patients with surgical need is great and it is a priority to identify those who would benefit from conservative management.

Keywords: Casuistry major chest trauma, Conservative management of chest trauma, Penetrating chest trauma.

\section{INTRODUCCION}

El tórax es la parte superior del tronco, su límite inferiores es el diafragma, el opérculo torácico superiormente y entre ambos la caja torácica; son varios los órganos vitales que se ubican completamente dentro de la cavidad torácica (corazón y pulmones) y otra parte recorren la cavidad torácica hacia el abdomen (aorta, esófago). ${ }^{1}$ Este corresponde a la cuarta parte de la masa corporal por lo que es susceptible a sufrir lesiones durante un trauma

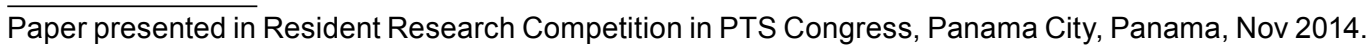


de cualquier etiología. ${ }^{2}$ El Trauma es conocido como una causa principal de muerte en los primeras cuatro décadas de la vida ${ }^{3}$ El primer documento referente a traumatismo torácico data de hace 5000 años atrás en el Papiro Quirúrgico de Edwin Smith escrito en el antiguo Egipto por Imhotep ${ }^{4}$ En la Iliada durante la guerra de Troya, Homero describe varias lesiones torácicas, el más conocido fue el del rey Agamenón, el cual asesinó a Odius durante la batalla de Troya con una lanza a través de su tórax ${ }^{5}$ Galeno describe las lesiones torácicas fatales sufridas por los antiguos atletas Olímpicos ${ }^{6}$ Durante la Segunda Guerra Mundial la toracotomía se utilizó como el medio más eficaz para drenar sangre retenida y desechos infectados. En los próximos decenios los avances en intubación endotraqueal, ventilación mecánica y control del dolor torácico permitió un adecuado manejo de las lesiones torácicass ${ }^{7,8}$ Durante la Guerra de Crimea entre los años de 1853 y 1856 la mortalidad por lesiones torácicas era del $28.5 \%$, hoy en día esta cifra ha descendido hasta un $5 \%{ }^{9}$ El 25\% de las muertes traumáticas son secundarios a lesiones torácicas, en pacientes con trauma torácico penetrante el $40 \%$ tiene lesión torácica, mientras que con un traumatismo cerrado el 33\% tienen una lesión torácica. ${ }^{10,11} \mathrm{El}$ traumatismo torácico representa uno de los mayores desafíos en el manejo integral del paciente politraumatizado debido a la alta morbilidad y mortalidad generada por sus complicaciones y los altos costos de la atención de salud. ${ }^{12}$ La mayoría de las lesiones torácicas son manejadas con maniobras simples como el tubo de toracostomía, apenas el 10 al 15\% de los pacientes necesitan tratamiento quirúrgico. ${ }^{13,14} \mathrm{El}$ hemoneumotórax es la lesion màs comúnmente encontrada en el trauma de tórax con una prevalencia del $52.3 \%$, seguido por el Hemotórax 23.4\% y el neumotórax del 20-20.7\% de los casos. ${ }^{15,16}$ Hasta el $30-80 \%$ de los pacientes con lesiones traqueo-bronquiales fallecen en el lugar del accidente. Actualmente, la incidencia de este tipo de lesiones entre los pacientes de trauma de tórax y cervical se estima en el 0,5-2\%. ${ }^{17}$ La Lesión aórtica se presenta en el 1,5 al 1,9\% de los accidentes automovilísticos y produce hasta el $15 \%$ de las muertes por esta etiología ${ }^{18-20}$ Las Lesiones cardíacas pueden implicar las lesiones en el miocardio, arterias coronarias, válvulas o el tabique con una mortalidad desde 10 al 70\%. ${ }^{21-24}$ Las lesiones de los grandes vasos torácicos son raras después de trauma torácico penetrante, con una incidencia del $4 \% .{ }^{25}$ Muchas de las lesiones de grandes vasos por trauma penetrante son exanguinantes antes de llegar a un hospital, con la mejora de la atención pre hospitalaria y la disminución de los tiempos de tránsito estas lesiones se encuentran cada vez más en centros de trauma. ${ }^{26}$ En las lesiones pulmonares del 20 al $40 \%$ de los pacientes con trauma penetrante y del 15 al 20\% de los pacientes con trauma cerrado requieren toracotomía con resección pulmonar, la mayoría de estas lesiones se resuelven con la colocación de un tubo de toracostomía, necesita algún tipo de resección pulmonar y hasta un $0.5 \%$ requieren de una resección pulmonar anatómica. ${ }^{27-29}$ Las lesiones del esófago intratorácico representan el 1\% de los traumatismos torácicos por arma de fuego. ${ }^{30}$

\section{MATERIALES Y METODOS}

Hicimos una recopilación en el registro de trauma de la sociedad panamericana de trauma localizado en la ciudad de Cali, tomando en cuenta a todos los pacientes que llegaron al HUV en el periodo de 01 de enero del 2012 hasta 31 de diciembre del 2013 con lesiones penetrantes de tórax y se revisó quienes tenían indicación quirúrgica y quienes requerían manejo conservador para de allí tener un dato estadístico, compararlo con series de casos de otros centros y establecer si es posible manejar conservadoramente a un porcentaje de pacientes que aparentemente son quirúrgicos.

\section{RESULTADOS}

Este estudio de colección retrospectiva de datos incluyo a los pacientes del registro de trauma, que sufrieron trauma penetrante de tórax en un periodo de 2 años y que fueron atendidos en el hospital universitario del valle, encontrándose 587 (Gráfico 1) pacientes con trauma penetrante de tórax, con un total de pacientes operados 257 (Gráfico 2) de los cuales 224 fueron dados de alta y 33 fallecieron; de los 330 (Gráfico 3) pacientes que no fueron operados 262 egresaron y 62 murieron. La mayoría eran hombres 557 y mujeres 30; con 2 tipos de heridas 247 punzocortantes y 340 heridas por proyectil de arma de fuego (Gráficos 4 and 5).

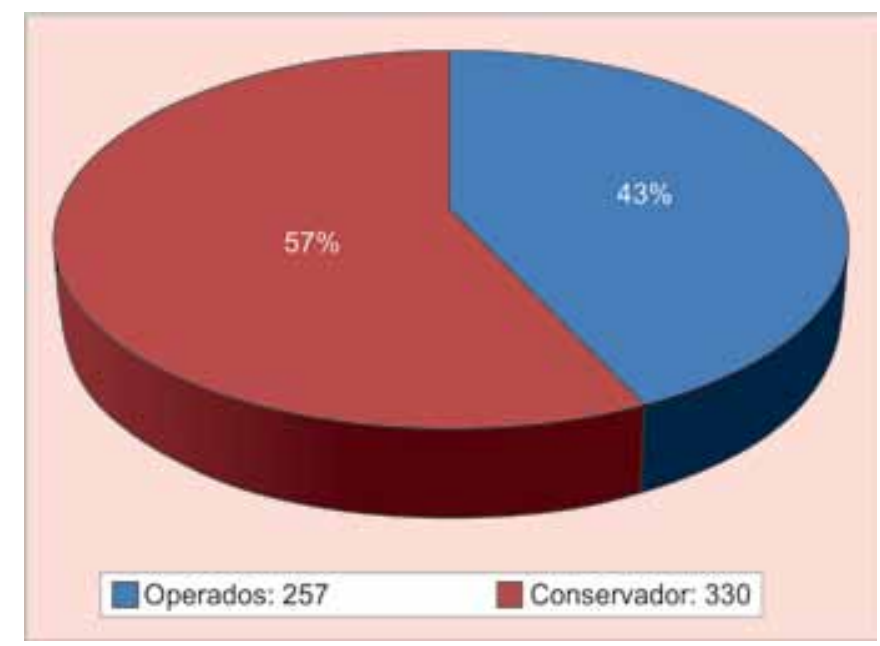

Gráfico 1: Total de 587 pacientes 


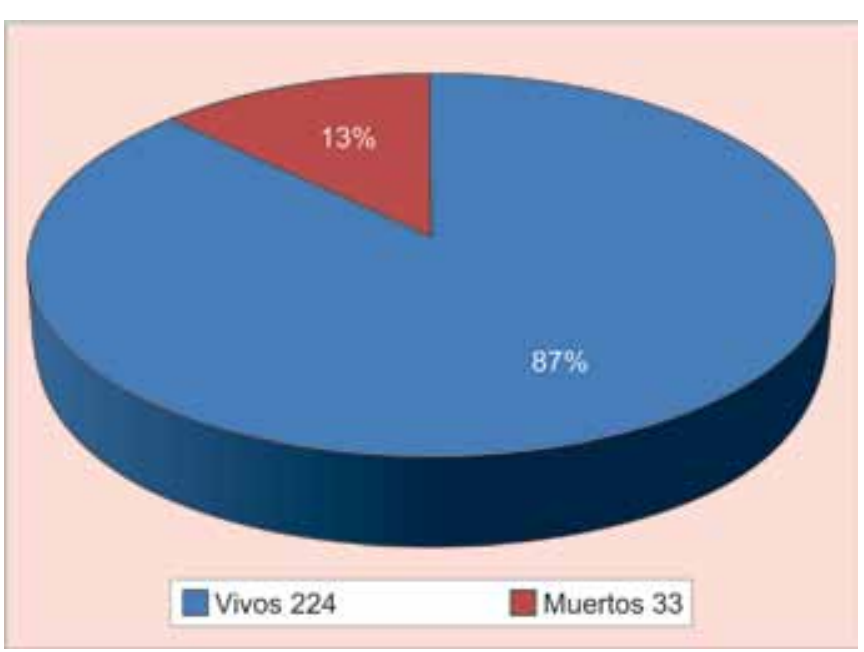

Gráfico 2: Pacientes operados: 257

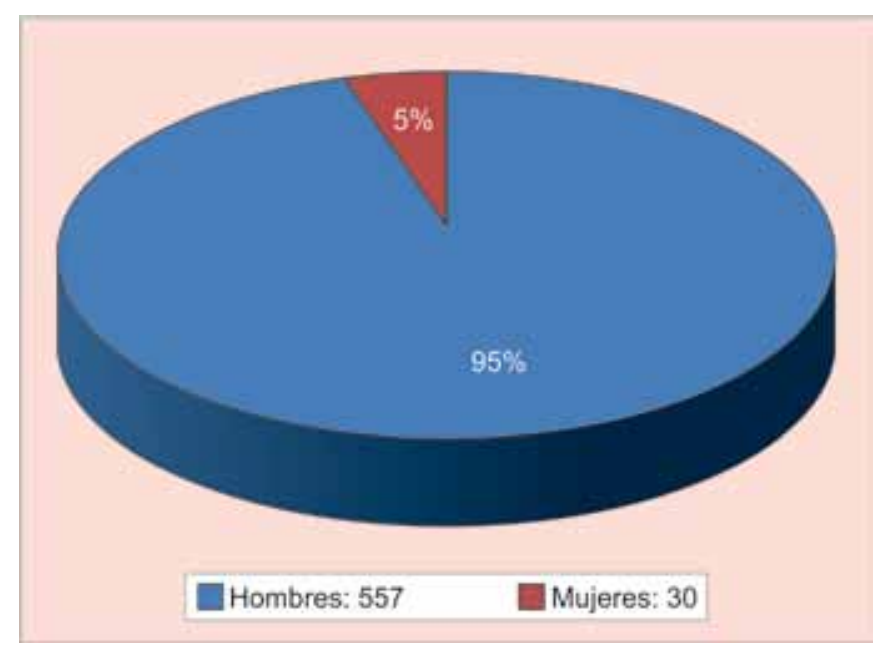

Gráfico 4: Sexo: pacientes 587

\section{DISCUSION}

El trauma penetrante de tórax fue definido como lesiones principalmente leves q en su gran mayoría se resuelven con procedimientos menores, los pocos casos complejos, menos del $15 \%$, requieren cirugía y tienen alta morbilidad y mortalidad, en nuestra recolección de datos pudimos evidenciar que los porcentajes están casi equiparados entre sí por lo que no guarda relación con las series conocidas, entonces podemos concluir que nuestro grupo de pacientes en primer lugar presentan lesiones más graves y más numerosas, en segundo lugar que las lesiones son de etiología intencional y casi ninguna accidental, lo que explica el elevado número de procedimientos y la alta mortalidad, sin embargo la observación diaria ha dejado una sensación de que algunos de los pacientes sometidos a cirugía de urgencia pudieron ser manejados de forma conservadora, lo que reduciría mortalidad, morbilidad y costos. Así que creemos que se deben plantear nuevos protocolos que incluyan esas variables.

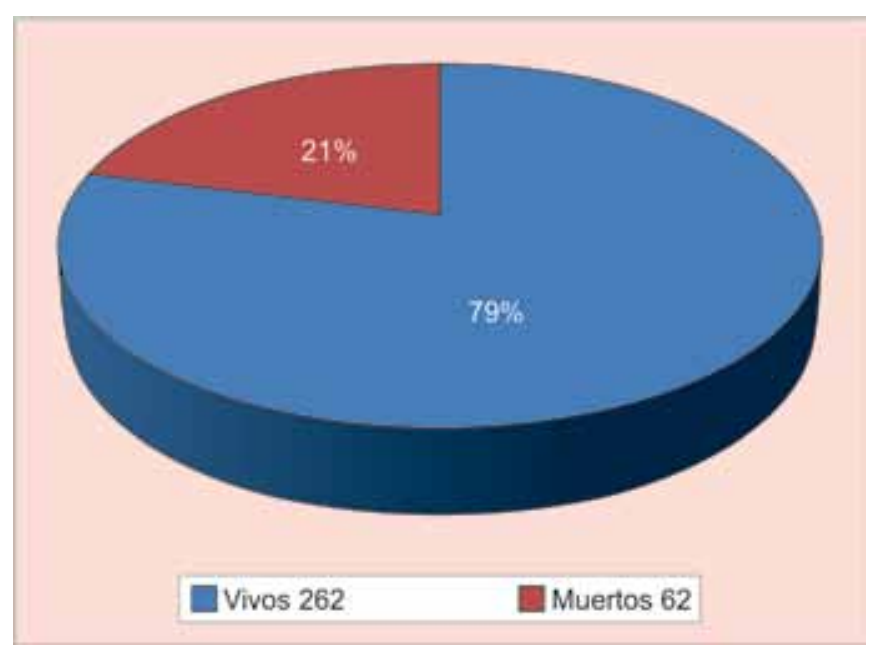

Gráfico 3: No operados: 324

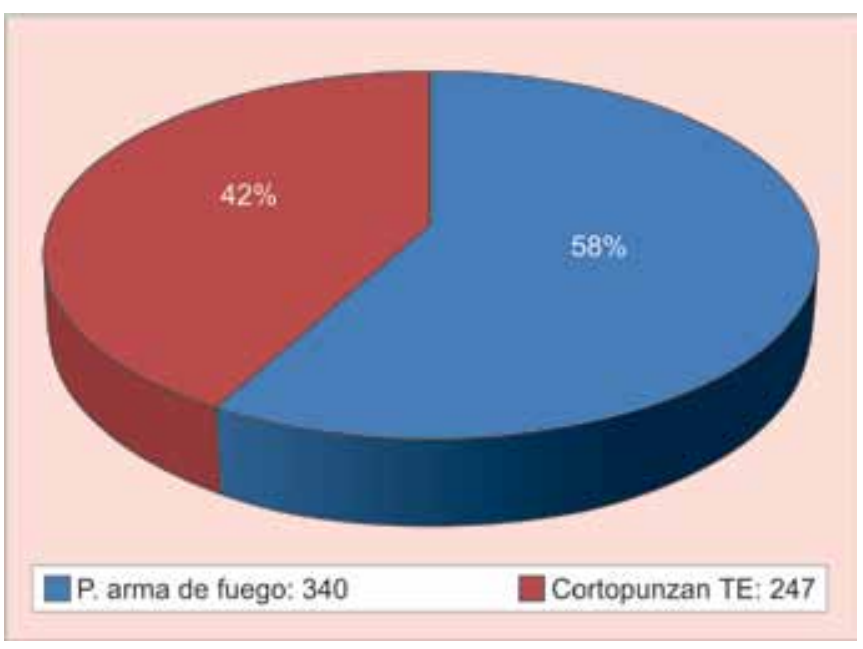

Gráfico 5: Tipo de arma: 587

\section{REFERENCIAS}

1. Sabiston and Spencer surgery of the chest. - 8th ed. / editor- in-chief, Frank W. Sellke; editors, Pedro J del Nido, Scott J. Swanson. by Saunders, an imprint of Elsevier Inc.; 2010.

2. Mattox K, Wall M, Tsai P. Trauma Thoracotomy: Principles and Techniques. In: Mattox K, Moore E, Feliciano D, editors. Trauma. 7th ed. New York: The McGraw-Hill Companies, Inc.; 2013. p. 461-467.

3. Batistella F, Benfield J. Blunt and penetrating injuries of the chest wall, pleura and lungs In: Shields TW. General Thoracic Surgery, 4th Ed. 1994;64:767-783.

4. Breasted JH. The Edwin Smith Surgical Papyrus. Chicago: University of Chicago Press; 1930;1.

5. Santos GH. Chest trauma during the battle of Troy: ancient warfare and chest trauma. Ann Thorac Surg 2000;69:1285-1287.

6. Menenakos E, Alexakis N, Leandros E, Laskaratos G, Nikiteas N, Bramis J, Fingerhut A. Fatal chest injury with lung evisceration during athletic games in ancient Greece. World J Surg 2005;29(10):1348-1351.

7. Asensio JA, Stewart BM, Murray J, Fox AH, Falabella A, Gomez H, et al. Penetrating cardiac injuries. Surg Clin North Am 1996;76(4):685-724. 
8. Sabiston and Spencer surgery of the chest. 8th ed. In: Nido PJ, Swanson, editors. By Saunders: an Imprint of Elsevier Inc.; 2010.

9. LoCicero J, Mattox KL. Epidemiology of chest trauma. Surg Clin North Am 1989;69:15.

10. Lee R. Traumatic injury of the cervicothoracic trachea and major bronchi. Chest Surg Clin N Am 1997;7:285-305.

11. Feliciano D. The diagnostic and therapeutic approach to chest trauma. Semin Thorac Cardiovasc Surg 1992;4:156-162.

12. Morales C, Mejìa C, Roldan L, Saldarriaga MF, Duque AF. Negative pleural suction in thoracic trauma patients: a randomized controlled trial. J Trauma Acute Care Surg 2014; 77:251-255.

13. Cohn S. Pulmonary contusion: review of the clinical entity. J Trauma 1997;42:973-979.

14. Hines M, Meredith J. Special problems of thoracic trauma. In: Ritchie WP, Steele G Jr, Dean RH, editors. General surgery. Philadelphia: JB Lippincott; 1995. p. 859-872.

15. Bartolomeo DS, Sanson G, Nardi G, Scian F, Michelutto V, Lattuada L. A population-based study on pneumothorax in severely traumatized patients. J Trauma 2001;51:677-682.

16. Villegas M,Hannessey R, Morales C, Londoño E. Risks factors associated with the development of post-traumatic retained hemothorax. Eur J Trauma Emerg Surg 2011;37:583-589.

17. Johnson S. Tracheobronchial injury. Semin Thorac Cardiovasc Surg 2008;20:52-57.

18. Fitzharris M, Frankly M, Frampton R, et al. Thoracic aortic injury in motor vehicle crashes: the effect of impact direction, side of body struck, and seat belt use. J Trauma 2004;57: 582-590.

19. Fabian T, Richardson J, Croce M, et al. Prospective study of blunt aortic injury: multicenter trial of the American
Association for the Surgery of Trauma. J Trauma 1997;42: 374-380.

20. McGwin G Jr, Reiff DA, Morgan SG, et al. Incidence and characteristics of motor vehicle collision-related blunt thoracic aortic injury according to age. J Trauma 2002;52: 859-865.

21. Demetriades D. Cardiac wounds: experience with 70 patients. Ann Surg 1986;203:315-317.

22. Honigman B, Rohweder K, Moore EE, et al. Prehospital advanced trauma life support for penetrating cardiac wounds. Ann Emerg Med 1990;19:145-150.

23. Demetriades $D, C$ Charalambides C, Sareli P, et al. Late sequelae of penetrating cardiac injuries. Br J Surg 1990;77:813-814.

24. Henderson V, Smith R, Fry W, et al. Cardiac injuries: analysis of an unselected series of 251 cases. J Trauma 1994;36:341-348.

25. Demetriades D. Penetrating injuries to the thoracic great vessels. J Card Surg 1997;12:173-179.

26. Honigman B, Rohweder K, Moore EE, et al. Prehospital advanced trauma life support for penetrating cardiac wounds. Ann Emerg Med 1990;19:145-150.

27. Karmy-Jones R, Jurkovich GJ, Shatz DV, et al. Management of traumatic lung injury: a Western Trauma Association Multicenter review. J Trauma 2001;51:1049-1053.

28. Borlase BC, Metcalf RK, Moore EE, et al. Penetrating wounds to the anterior chest: analysis of thoracotomy and laparotomy. Am J Surg 1986;152:649-653.

29. Thompson DA, Rowlands BJ, Walker WE, et al. Urgent thoracotomy for pulmonary or tracheobronchial injury. J Trauma 1988;28:276-280.

30. Cornwell EE III, Kennedy F, Ayad IA, et al. Transmediastinal gunshot wounds: a reconsideration of the role of aortography. Arch Surg 1996;131:949-952. 


\section{Manejo Conservador del Trauma Penetrante de Tórax, Casuística de 2 Años en Hospital Universitario del Valle en Cali, Colombia, un Importante Centro de Trauma}

En este interesante trabajo los autores presentan un estudio retrospectivo del manejo Conservador del Trauma Torácico en el Hospital del Valle, en Cali Colombia, desde el 1 de enero del 2012 hasta el 31 de diciembre del 2013.

Las variables analizadas fueron: tratamiento quirúrgico y conservador, mortalidad y sobrevida en ambos grupos, sexo y mecanismo de lesión.

Se presentaron 587 pacientes con trauma penetrante de tórax. La mayoría eran de sexo masculino $(94,89 \%)$. Las heridas por arma de fuego representaron el 58\% de los casos. Del total de pacientes, el 43,58\% (257) fueron sometidos a tratamiento quirúrgico y el 56,22\% (330) fueron sometidos a tratamiento conservador. Este reporte llamó la atención de los autores del trabajo y lo resaltan ya que es un hallazgo que no concuerda con la mayoría de las estadísticas internacionales, donde el tratamiento quirúrgico del trauma torácico penetrante es menor del 15\%, como ellos mismos lo concluyen.

Considero que para llegar a algunas de las opiniones que los autores tratan de emitir para explicar esta casuística tan baja de tratamiento conservador, faltaron variables por presentar como por ejemplo, los hallazgos operatorios, especialmente en el grupo de pacientes vivos, con lo cual podríamos discutir las indicaciones de tratamiento quirúrgico en este grupo de pacientes. 


\section{Conservative Management of Penetrating Trauma of Thorax: 2 Years Experience in Hospital Universitario del Valle in Cali, Colombia-An Important Center of Trauma Management}

In this interesting work, the authors present a retrospective study Conservative management of chest trauma at the Hospital del Valle in Cali, Colombia, from 1 January 2012 until 31 December 2013.

The variables analyzed were: surgical and conservative treatment, mortality and survival in groups, sex and injury mechanism.

A total of 587 patients presented with penetrating chest trauma. Most were male (94.89\%) sex. The gunshot wounds accounted for $58 \%$ of cases. Out of the total of patients, $43.58 \%$ (257) were submitted to surgical treatment and $56.22 \%$ (330) underwent conservative treatment. This report caught the attention of the authors of the work and highlight as it is a finding that is not consistent with most international statistics, where the surgical treatment of penetrating chest trauma is less than $15 \%$, as they themselves conclude.

I belive that the authors should submit missing variables, such as operative findings, especially in survivors and indications for surgical treatment to explain the low incidence of conservative treatment.

Jorge Rabat S

Jefe Departamento de Cirugía

Universidad de Oriente

Hospital Ruiz y Paez, Bolívar, Venezuela 